\title{
RDUS
}

Revue de DROIT

UNIVERSITÉ DE SHERBROOKE

Titre : $\quad$ LES CONFLITS D'INTÉRÊTS INSTITUTIONNELS AU SEIN DE LA MAGISTRATURE

Auteur(s): $\quad$ LUc HUPPÉ

Revue : $\quad$ RDUS, 2007-2008, volume 38, numéro 1

Pages: $\quad 127-165$

ISSN : $\quad 0317-9656$

Éditeur : Université de Sherbrooke. Faculté de droit.

URI : $\quad$ http://hdl.handle.net/11143/11561

DOI : https://doi.org/10.17118/11143/11561 
Page vide laissée intentionnellement. 


\title{
ARTICLE
}

\section{LES CONFLITS D'INTÉRÊTS INSTITUTIONNELS AU SEIN DE LA MAGISTRATURE}

\author{
par Luc HUPPÉ*
}

Le principe de l'impartialité judiciaire exige la récusation d'un juge placé en conflit d'intérêts. En invoquant la nécessité, la jurisprudence a élaboré une exception à cette obligation lorsque tous les juges du tribunal se trouvent dans cette situation conflictuelle. Cette doctrine de la nécessité a notamment été utilisée pour permettre à un tribunal de trancher des litiges concernant la rémunération des juges ou encore les ressources mises à leur disposition pour l'exercice de leurs fonctions judiciaires. Dans une première partie, le présent article passe en revue les jugements rendus dans de telles circonstances afin d'examiner l'utilisation que les tribunaux font de la doctrine de la nécessité pour justifier leur compétence. La seconde partie analyse les fondements de cette doctrine et en propose diverses modalités d'application.

Judicial impartiality requires the disqualification of a judge who is in a conflict of interest. However, the doctrine of necessity allows a judge to hear a case when all the judges of a same court would be disqualified. The doctrine of necessity was used, for instance, in cases involving matters related to the remuneration of judges or the allocation of resources incident to their office. The first part of this article contains a review of the cases where the doctrine of necessity served to solve a conflict of interest of this nature. The second parts contains an analysis of the principles underlying the doctrine of necessity and suggests various ways in which it may be applied.

\footnotetext{
* Avocat, Lafortune Cadieux, Montréal.
} 


\section{SOMMAIRE}

INTRODUCTION

\section{LE TRAITEMENT DES CONFLITS D'INTÉRÊTS}

INSTITUTIONNELS ...................................... 132

1.1 Les intérêts pécuniaires des juges ................... 132

1.2 Les autres situations de conflit d'intérêts ........... 139

2. LA DOCTRINE DE LA NÉCESSITÉ ........................ 149

$2.1 \quad$ Les fondements de la doctrine ........................ 149

2.2 Les modalités de la doctrine ........................... 159

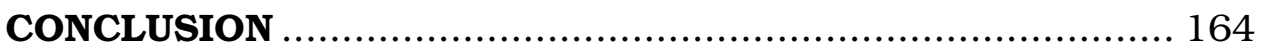




\section{INTRODUCTION}

Le Code de procédure civile énonce différentes situations de conflits d'intérêts susceptibles d'entraîner la récusation d'un juge à l'égard d'un litige dont il est saisi ${ }^{1}$. La plupart des motifs de récusation découlent des relations personnelles, familiales ou professionnelles du juge avec une partie au litige. C'est le cas, par exemple, lorsque le juge est parent de l'une des parties ou de son procureur, si le juge a agi comme avocat pour l'une des parties, ou encore s'il est membre d'une association partie au litige. De façon générale, la récusation est aussi possible lorsque le juge "a quelque intérêt à favoriser l'une des parties", ou encore "s'il existe une crainte raisonnable que le juge puisse être partial»'2. Ces dispositions servent de toute évidence à préserver l'impartialité réelle et apparente du tribunal et la common law contient des principes similaires, visant le même objectif ${ }^{3}$.

Des motifs de récusation personnels à un juge ne rendent pas inhabiles les autres membres du tribunal. Ce principe vaut tant au sein d'un tribunal d'appel, où les juges siègent en collégialité4, que dans un tribunal de première instance, où les juges siègent de façon indépendante les uns des autres ${ }^{5}$ Ainsi, la récusation d'un juge n'empêche-t-elle pas le tribunal dont il est

1. $\quad$ Code de procédure civile, L.R.Q. c. C-25, art. 234-235.

2. Ibid., art. 234 (8), (10).

3. John O. Wilson, A Book for Judges, Ottawa, Ministre des approvisionnements et services du Canada, 1980 à la p. 23 et s.; Philip Bryden, "Legal Principles Governing the Disqualification of Judges", (2003) 82 R. du B. can. 555. En 1998, le Conseil canadien de la magistrature écrivait que le Code de procédure civile du Québec "est la seule source législative qui fasse autorité en la matière au Canada": Conseil canadien de la magistrature, Principes de déontologie judiciaire, Ottawa, 1998 au para. E.4, à la p. 43 [Conseil canadien de la magistrature].

4. Mugesera c. Canada (Ministre de la Citoyenneté et de l'Immigration), [2005] 2 R.C.S. 91 au para. 15. Voir aussi Bande indienne Wewaykum c. Canada, [2003] 2 R.C.S. 259 au para. 93.

5. Gillet c. Arthur, [2005] R.J.Q. 42 au para. 37(C.A.). Autorisation de pourvoi refusée, [2005] 1 R.C.S. vi. 
membre d'entendre et de décider du litige en toute impartialité. Par ailleurs, le simple fait qu'un juge soit lui-même partie à un litige d'ordre privé n'empêche pas le tribunal qui en est saisi d'exercer sa compétence et ce, même lorsque le juge partie au litige est membre d'un tribunal hiérarchiquement plus élevé que celui appelé à le trancher6.

En théorie, le mécanisme de récusation devrait recevoir application à l'ensemble des litiges quand survient une situation de conflit d'intérêts. Son application pose problème lorsqu'un tribunal est saisi de litiges qui concernent les membres de la magistrature en leur qualité de juges, plutôt qu'en leur qualité personnelle; une telle situation se produit, par exemple, quand un tribunal est saisi d'un litige relatif à la rémunération de ses membres. Il existe alors un conflit d'intérêts institutionnel, c'est-à-dire une situation où l'intérêt du juge dans le litige ne provient pas de considérations propres à sa personne mais découle plutôt de la fonction qu'il occupe.

L'intérêt personnel d'un juge dans ce type de litiges peut prendre deux formes. En premier lieu, les conclusions du jugement que le juge est appelé à rendre peuvent produire un effet direct à son égard, en sa qualité de juge : ce serait par exemple le cas si le juge devait apprécier la validité des dispositions qui fixent sa rémunération et celle de ses collègues. En second lieu, bien qu'il ne soit pas directement visé par les conclusions de son jugement, les principes de droit que le juge formule dans le cadre de son jugement peuvent lui procurer un certain bénéfice, en sa qualité de juge : par exemple, s’il apprécie la validité constitutionnelle des dispositions qui fixent la rémunération des membres d'un tribunal autre que celui dont il fait partie, il pourrait éventuellement bénéficier de certains principes de droit utilisés dans son juge-

6. À titre d'exemple, la Cour supérieure a rejeté l'allégation d'apparence de partialité dont elle était l'objet à l'égard des procédures de divorce impliquant un juge de la Cour d'appel et s'est déclarée compétente à entendre le litige : A. c. B., [2006] R.J.Q. 2446 (C.S.). L'article 234 (2) du Code de procédure civile prévoit cependant que la récusation est possible si le juge est partie à un procès portant sur une question pareille à celle dont il est saisi. 
ment, dans l'hypothèse où surgissait ultérieurement un litige relié à sa propre rémunération.

Dans de telles situations, l'application des règles qui régissent la récusation des juges conduit à un cul-de-sac. S'il pouvait être invoqué avec succès, le motif de récusation relié à l'intérêt personnel du juge saisi du litige rendrait inhabiles l'ensemble des membres du tribunal. En effet le même conflit d'intérêts existe alors pour tous les juges du tribunal, puisque c'est précisément leur qualité de juge qui les rend intéressés au litige. La jurisprudence canadienne a parfois résolu ce problème en s'inspirant d'une "doctrine de la nécessité", développée en droit britannique à l'égard de diverses situations de conflit d'intérêts et qui écarte l'application des règles relatives à la récusation. Selon cette doctrine, lorsque tous les juges disponibles d'un tribunal ont un intérêt dans un litige, l'un ou l'autre d'entre eux peut néanmoins en disposer.

Au cours des dernières décennies, les juges canadiens ont commencé à exercer avec une plus grande fréquence les droits rattachés à leur fonction. Alors qu'elles ne se présentaient auparavant que de façon exceptionnelle ${ }^{7}$, les situations de conflit d'intérêts institutionnel surgissent maintenant plus souvent, bien que le nombre de jugements rendus dans un tel contexte soit encore relativement restreint. Par voie de conséquence, la doctrine de la nécessité trouve plus régulièrement application à l'égard de tels conflits d'intérêts. Pourtant, cette doctrine n'a pas encore fait l'objet d'une véritable analyse. Il en résulte qu'un principe élaboré sommairement par la jurisprudence pour régler des situations exceptionnelles est dorénavant reçu, sans autre examen, comme

7. En ce qui concerne les conflits d'intérêts institutionnels au sein de la magistrature, la doctrine de la nécessité n’a trouvé application, jusqu'au début des années 1980, que dans un nombre restreint de litiges, essentiellement reliés à la rémunération des juges : R.R.S. Tracey, "Disqualified Adjudicators : The Doctrine of Necessity in Public Law", [1982] P.L. 628 aux pp. 631-632 [R.R.S. Tracey]. Thomas Mckevitt, "The Rule of Necessity : Is Judicial Non-Disqualification Really Necessary ?", (1995-1996) 24 Hofstra L. Rev. 817 aux pp. 830-834. 
une règle de droit bien établie. Pour en permettre une application adéquate, respectueuse $\mathrm{du}$ contexte juridique canadien, il convient donc d'examiner la justification et la portée de cette doctrine.

Une remarque préliminaire s'impose. La question des conflits d'intérêts institutionnels au sein de la magistrature est délicate. Le fait qu'un juge se trouve placé dans une telle situation ne l'empêche pas nécessairement de rendre, avec discernement, un jugement pondéré à tous points de vue. On ne peut négliger les efforts sincères et réels que démontrent les juges placés malgré eux dans cette position, afin d'en arriver à une décision juste et équitable. Il ne saurait donc être question ici de remettre en cause le bien-fondé des jugements rendus dans un tel contexte, ni d'attaquer l'impartialité réelle de leurs auteurs. L'objet du présent texte est plutôt d'analyser la façon dont les tribunaux traitent ces situations de conflit d'intérêts institutionnel, ainsi que leur utilisation de la doctrine de la nécessité à cette fin.

\section{LE TRAITEMENT DES CONFLITS D'INTÉRÊTS INSTITU- TIONNELS}

De nombreux sujets peuvent susciter un conflit d'intérêts institutionnel au sein de la magistrature, quoique tous les litiges portant sur de tels sujets ne donnent pas nécessairement lieu à une analyse du conflit d'intérêts. Les litiges reliés aux intérêts pécuniaires des juges présentent un cas classique d'application de la doctrine de la nécessité, qui a aussi été invoquée à l'égard d'autres facettes de la fonction judiciaire.

\subsection{Les intérêts pécuniaires des juges}

C'est une situation mettant en cause les intérêts pécuniaires des juges qui donne lieu, au milieu des années 1930, au premier jugement traitant d'un conflit d'intérêts institutionnel en droit canadien. Au moyen d'un renvoi, le gouvernement de la Saskatchewan demande l'opinion de la Cour d'appel de la province quant à l'autorité de la législature provinciale d'assujettir à un 
impôt sur le revenu les traitements des juges nommés et rémunérés par le gouvernement fédéral. Les juges de la Cour d'appel de la Saskatchewan sont ainsi appelés à déterminer, entre autres, si leur propre rémunération est imposable. Ils abordent d'emblée la question de leur intérêt dans le litige :

It is a fundamental rule in the administration of justice that where a Judge is interested in the result of litigation he cannot sit in judgment upon it. Nemo debet esse judex in propria causa. Proceedings have been frequently set aside because a Judge who had an interest in the cause took part in the decision. According to the rule, therefore, the members of the Court should not participate in the reference, because each of us has a pecuniary interest in the result. The rule, however, does not apply where the Court acts ex necessitate, e.g., where an action is brought against all the Judges of the Court in a matter over which the Court has exclusive jurisdiction (...). ${ }^{8}$

De toute évidence, le fait que le gouvernement de la province ait pris l'initiative de ce renvoi facilite l'application de la doctrine de la nécessité par le tribunal :

The present reference has been made to the Court of Appeal under the provisions of the Constitutional Questions Act, and the Court is required not only to hear and consider the subject referred, but also to certify its opinion to the Lieutenant-Governor in council and to give reasons therefore. Moreover, all Judges of the Province have the same interest in the result of the reference as have the members of this Court. The Court, therefore, acts ex necessitate. ${ }^{9}$

Ceci étant, la Cour d'appel de la Saskatchewan conclut que les juges de nomination fédérale peuvent effectivement être assujettis à un impôt sur le revenu par la législature provinciale. Porté

8. Re The Constitutional Questions Act, [1936] 4 D.L.R. 134 à la p. 135 (C.A. Sask.).

9. Ibid. 
en appel, cet arrêt est confirmé par le Comité judiciaire du Conseil privé, composé de juges qui ne sont pas visés par les dispositions législatives faisant l'objet du renvoi. À propos de la situation de conflit d'intérêts dans laquelle se trouvaient les juges de la Cour d'appel, le Comité judiciaire confirme le bien-fondé de la position prise par ceux-ci :

The reference in question placed the Court in an embarrassing position, all its members being from the nature of the case personally interested in the point in controversy. They took the view (quite rightly in their Lordships' opinion) that they were bound to act ex necessitate. In the result they came unanimously to a conclusion adverse to the contention put before them on behalf of their order. ${ }^{10}$

Cette première affaire présente donc une application peu discutable de la doctrine de la nécessité, et ce, pour plusieurs raisons. Ce ne sont pas les membres de la magistrature qui prennent l'initiative du litige, mais le gouvernement provincial. Il n'existe dans la province qu'un seul tribunal pouvant être saisi du renvoi. Les juges de la Cour d'appel divulguent expressément leur intérêt dans le litige, ainsi que l'obligation qui leur est faite par la loi de répondre aux questions qui leur sont soumises par le gouvernement. D'aucune façon leur impartialité réelle ne peut-elle être mise en doute, notamment en raison du fait que la conclusion à laquelle en arrive le tribunal est contraire aux intérêts économiques des juges concernés par le renvoi. Enfin, l'arrêt fait l'objet d'un appel auprès d'un tribunal composé de membres dont l'impartialité ne peut être affectée par la nature du litige.

L'obligation des juges de nomination fédérale de cotiser à un fonds de pension ${ }^{11}$ à même le revenu qui leur est versé par le

10. The Judges v. Attorney-General for Saskatchewan, [1937] 1 W.W.R. 508 à la p. 509 (P.C.) [The Judges].

11. Le droit d'un juge de nomination fédérale d'obtenir une pension fait aussi l'objet d'un litige à la même époque : Landreville c. La Reine, [1981] 1 C.F. 15 (C.F. 1 1 re inst.). Le juge saisi de ce litige devait interpréter la disposition législative fixant les conditions permettant à un juge de nomination fédérale d'obtenir une pension. Cette disposition lui était aussi vraisemblable- 
gouvernement fédéral donne lieu, cinquante ans plus tard, à une autre situation de conflit d'intérêts institutionnel. Cette fois, c'est un juge qui prend l'initiative du litige. Nommé à la Cour supérieure du Québec avant l'adoption des dispositions législatives imposant cette obligation, mais postérieurement à leur date d'entrée en vigueur rétroactive, il en conteste la validité devant la Cour fédérale du Canada. Cette dernière est également composée de juges visés par ces dispositions. La situation de conflit d'intérêts institutionnel dans laquelle se trouvent les juges appelés à se prononcer à propos de ce litige reçoit un traitement différent par chacune des instances qui en sont successivement saisies.

En première instance, le jugement contient une discussion détaillée de la question, de même qu'une divulgation par le juge de l'étendue de son intérêt dans le litige ${ }^{12}$. La doctrine de la nécessité est ensuite invoquée pour asseoir la compétence du tribunal à trancher le litige :

...lorsqu'il se pose une importante question de droit dans laquelle tous les juges ont un intérêt personnel, elle peut être tranchée par un juge sur la base de la nécessité, le raisonnement étant que, dans pareil cas, on présume que la justice est mieux servie en faisant trancher la question

ment applicable, bien qu'il ne se trouvait pas dans la même situation que le juge ayant initié le litige. La question d'un potentiel conflit d'intérêts n'est pas traitée dans le jugement. Cette question n'a pas non plus été abordée lorsque des juges ont été appelés à déterminer, à l'égard de certains de leurs collègues, si les sommes découlant du régime de pension des juges de nomination fédérale font partie du patrimoine familial devant être séparé entre les époux au cas de divorce : Droit de la famille-1473, [1991] R.D.F. 524 (C.S.); Droit de la famille-2743, [1997] R.J.Q. 2134 (C.A.).

12. Beauregard c. La Reine, [1981] 2 C.F. 543 aux pp. 549-551 (C.F. 1re inst.) [Beauregard]. Nommé antérieurement à la date d'entrée en vigueur des modifications législatives qui donnent lieu à la contestation, il ne se trouve donc pas dans la même situation que le juge ayant initié le litige. Toutefois, cette réforme lui impose aussi un certain fardeau financier, moindre que celui que doit supporter le juge qui a pris l'initiative des procédures. 
par quelqu'un qui a un intérêt personnel dans l'issue de l'affaire qu'en laissant la question en suspens. ${ }^{13}$

Au mérite du litige, la Cour fédérale de première instance donne raison au juge qui en a pris l'initiative et conclut à l'inconstitutionnalité des dispositions contestées. Devant la Cour d'appel fédérale, également composée de juges touchés par la réforme législative, la question du conflit d'intérêts se présente différemment: la Cour s'abstient d'en traiter en raison du consentement donné par les parties à la compétence du tribunal. L'un des juges prend cependant le soin de le mentionner spécifiquement dans ses motifs de jugement :

Si j'ai bien compris les avocats des deux parties, ces dernières ont convenu entre elles qu'il s'agit en l'espèce d'une question dont les tribunaux peuvent être saisis et qui doit être tranchée par des juges nommés par le fédéral et que même s'il est possible que lesdits juges aient potentiellement un intérêt identique ou similaire dans l'issue du litige, la Cour doit néanmoins instruire l'affaire ex necessitate. ${ }^{14}$

À la majorité, le jugement de première instance est confirmé par la Cour d'appel fédérale. Le litige aboutit à la Cour suprême du Canada ${ }^{15}$, qui rejette les procédures du juge ayant intenté les procédures et confirme la validité des dispositions législatives qu'il contestait. Bien que ses membres soient aussi visés par la réforme législative en cause, la Cour suprême du Canada n'aborde pas la question du conflit d'intérêts dans laquelle ceux-ci se trouvent. Dans un arrêt subséquent, où elle discute d'une autre application de la doctrine de la nécessité, elle confirmera toutefois que ce litige plaçait bel et bien les juges de la Cour en conflit d'intérêts :

13. Ibid. à la p. 551.

14. Beauregard c. La Reine, [1984] 1 C.F. 1010 à la p. 1038 (C.A.F.).

15. Beauregard c. Canada, [1986] 2 R.C.S. 56. 
Notre cour s'est fondée implicitement sur cette règle dans l'arrêt [Beauregard], où nous avons examiné la constitutionalité de dispositions exigeant que les juges nommés par le gouvernement fédéral, y compris les juges de notre Cour, contribuent une partie de leur traitement au paiement du coût des pensions. ${ }^{16}$

On constate donc dans cette affaire une atténuation graduelle de la préoccupation reliée au conflit d'intérêts, au fur et à mesure de la progression du litige. Le consentement donné par les parties à la compétence du tribunal explique sans doute le traitement différent de la situation par les diverses instances judiciaires. Un tel consentement ne règle pourtant pas la question de façon entièrement satisfaisante. Si les parties à un litige peuvent conduire leurs procédures comme elles l'entendent, afin d'obtenir un jugement qui détermine leurs droits et obligations, en revanche les principes formulés par le tribunal à l'occasion de ce litige produisent aussi des effets à l'égard des tiers, en raison de la valeur accordée au jugement à titre de précédent. Dans cette perspective, l'exigence d'une impartialité réelle et apparente qui s'impose au tribunal ne peut être amoindrie du simple consentement des parties à un litige. Tout au plus, ce consentement peut-il servir à conforter le tribunal qui, par ailleurs, en arrive à la conclusion que la doctrine de la nécessité doit recevoir application pour lui permettre de trancher le litige.

Au cours de la décennie suivante, les tribunaux supérieurs de plusieurs provinces sont saisis de nombreux litiges concernant la rémunération des juges de nomination provinciale. À l'origine, ces litiges ne présentent pas de conflits d'intérêts institutionnels, étant donné que les tribunaux qui en décident ne comptent parmi leurs membres aucun des juges dont la rémunération est en cause. Au niveau de la Cour suprême du Canada ${ }^{17}$, toutefois, les

16. Renvoi relatif à la rémunération des juges de la Cour provinciale (Î-P-É), [1998] 1 R.C.S. 3 au para. 5.

17. Renvoi: juges de la Cour provinciale, [1997] 3 R.C.S. 3. Association des juges de la Cour provinciale du Nouveau-Brunswick c. Nouveau-Brunswick 
principes dégagés à l'occasion de ces litiges profitent à l'ensemble de la magistrature canadienne. En définissant les exigences constitutionnelles relatives à la rémunération des juges de nomination provinciale, les membres de la Cour se trouvent indirectement à définir l'encadrement juridique requis pour établir celle des juges de nomination fédérale, y compris la leur. La question d'un possible conflit d'intérêts parmi ses membres n'est pourtant traitée dans aucun des arrêts prononcés par la Cour suprême du Canada à ce sujet. En tant qu'ultime instance d'appel au Canada, la Cour suprême est toutefois placée dans une position bien différente de celle des tribunaux qui lui sont hiérarchiquement subordonnés : aucune autre instance ne peut exercer une compétence semblable à la sienne ou réviser ses jugements. Plus que dans tout autre tribunal du pays, la doctrine de la nécessité peut trouver application à la Cour suprême du Canada sans qu'aucune explication particulière ne soit requise.

Les litiges mettant en cause la responsabilité civile des juges suscitent aussi des situations de conflit d'intérêts institutionnel. Sans qu'il n'existe d'assise législative en ce sens, la jurisprudence a développé au bénéfice des juges le principe d'une immunité absolue, qui les protège à l'égard des paroles prononcées et des actes accomplis dans l'exercice de leurs fonctions. En invoquant cette immunité pour rejeter l'action intentée contre l'un de ses collègues, un juge définit par voie de conséquence le cadre juridique de sa propre responsabilité civile. Pourtant, qu'elle concerne les juges des tribunaux supérieurs ${ }^{18}$ ou les juges des tribunaux inférieurs ${ }^{19}$, cette situation n'a pas été traitée par la

(Ministre de la justice), [2005] 2 R.C.S. 289. Voir aussi Mackin c. NouveauBrunswick (Ministre des Finances), [2002] 1 R.C.S. 405.

18. Bengle c. Weir, (1929) 67 C.S. 289. Royer c. Mignault, [1985] C.S. 1017, conf. par : [1988] R.J.Q. 670 (C.A.), autorisation de pourvoi à la C.S.C. refusée : [1988] 1 R.C.S. xiii. Taylor c. Canada (Procureur général), [2000] 3 C.F. 298 (C.A.F.). Le seul arrêt dans lequel la Cour suprême du Canada se soit prononcée à propos de l'immunité de poursuite des juges concernait non un membre de la magistrature, mais les membres d'une commission d'enquête : Morier c. Rivard, [1985] 2 R.C.S. 716 aux pp. 737-745.

19. Janson c. King, (1980) 103 D.L.R. (3rd) 189 (Q.B. Sask.). Charters c. Harper, (1987) 31 D.L.R. (4 ${ }^{\text {th }} 468$ (Q.B.N.-B.). 
jurisprudence sous l'angle d'un conflit d'intérêts. Il faut cependant constater que l'immunité de poursuite des juges est une règle de droit très ancienne, dont l'à-propos n'a jamais été remis en question par le législateur bien qu'elle ait été façonnée par ceux-là même qui en bénéficient.

\subsection{Les autres situations de conflit d'intérêts}

Plusieurs autres facettes de la fonction judiciaire sont aussi susceptibles de donner lieu à des conflits d'intérêts institutionnels. On peut regrouper en trois catégories les litiges dans lesquels ils se sont jusqu'à présent manifestés: ceux reliés au maintien du juge dans ses fonctions judiciaires; ceux ayant trait à l'exercice des droits civiques des juges; ceux concernant la gestion et la direction du tribunal.

N'étant pas nommément visés par les dispositions de la Loi constitutionnelle de 1867 qui permettent aux juges des tribunaux supérieurs d'occuper leur fonction jusqu'à l'âge de soixante-quinze ans $^{20}$, les juges de la Cour fédérale du Canada étaient jadis tenus par la loi de prendre leur retraite à l'âge de soixante-dix ans. Au début des années 1980, un juge de cette Cour s'adresse au tribunal dont il fait partie pour contester cette limite ${ }^{21}$. À cette occasion, la Cour fédérale du Canada doit ainsi déterminer la validité constitutionnelle d'une disposition législative qui concerne la mise à la retraite de ses propres membres. Ce litige place donc le juge qui en est saisi dans la position de devoir statuer à propos de sa propre inamovibilité. Toutefois, c'est un juge suppléant, et non un juge permanent de la Cour fédérale, qui entend le litige ${ }^{22}$, ce qui

20. Loi constitutionnelle de 1867 (R.-U.), 30 \& 31 Vict., c. 3, art. 99 (2), reproduit dans L.R.C. 1985, app. II, n5.

21. Addy c. R., [1985] 2 C.F. 452 (C. F. 1re inst.) [Addy]; il est intéressant de noter que le juge qui demande cette réparation est celui-là même qui, quelques années plus tôt, avait rendu le jugement de première instance dans l'affaire Beauregard, supra note 12.

22. Le gouverneur en conseil pouvait en effet autoriser le juge en chef de la Cour fédérale à demander l'affectation à ce tribunal de juges choisis parmi les juges en exercice ou les juges retraités d'une cour supérieure, d'une cour de comté ou d'une cour de district au Canada: Loi sur la Cour fédé- 
diminue le conflit d'intérêts et explique, sans doute, que la question ne soit pas abordée dans le jugement. Bien qu'il exerce les pouvoirs de la Cour fédérale et qu'il soit désigné par un juge assujetti à l'obligation de prendre sa retraite à l'âge de soixante-dix ans, le juge suppléant n'est pas visé par la disposition dont il doit apprécier la validité23. Il la déclare par ailleurs inconstitutionnelle et son jugement n'est pas porté en appel.

Les litiges relatifs à la déontologie et la discipline des juges, ou qui concernent le processus permettant de les destituer, peuvent aussi donner lieu à des situations de conflit d'intérêts institutionnel. En élaborant les principes applicables aux litiges concernant certains de leurs collègues, les juges qui en sont saisis se trouvent à déterminer le cadre juridique dans lequel ils sont eux-mêmes redevables de leur conduite. Les quelques rares jugements rendus en cette matière à propos de juges de nomination fédérale, depuis la création du Conseil canadien de la magistrature, n'ont pas abordé cette question ${ }^{24}$. Par ailleurs, en ce qui a trait à la discipline et à la destitution des juges de nomination provinciale, chaque province possède son propre mécanisme d'enquête à propos des plaintes formulées contre eux et ce sont les tribunaux supérieurs des provinces, dont les juges ne sont pas assujettis à ces mécanismes, qui contrôlent les décisions des conseils de la magistrature provinciaux ${ }^{25}$.

rale, S.R.C. 1970 (2ième supp.) c. 10, art. 10. Des dispositions semblables se trouvent maintenant à l'article 10 de la Loi sur les Cours fédérales, L.R.C. 1985, c. F-7. Ces juges obtiennent la qualité de juges suppléants et sont investis des pouvoirs des juges de la Cour fédérale.

23. En l'instance, le juge saisi du litige mentionne spécifiquement que la loi ne fixait aucune limite d'âge pour l'exercice de fonctions judiciaires par un juge suppléant : Addy, supra note 21 à la p. 464.

24. Par exemple : Gratton c. Conseil canadien de la magistrature, [1994] 2 C.F.

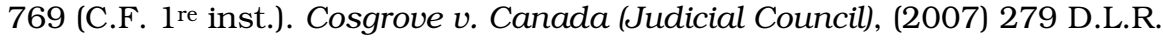
(4th) 352 (C.A.F.).

25. Pour des exemples de litiges concernant la discipline de juges de nomination provinciale, voir : Ruffo c. Conseil de la magistrature, [1995] 4 R.C.S. 267. Therrien (Re), [2001] 2 R.C.S. 3. Moreau-Bérubé c. Nouveau-Brunswick (Conseil de la magistrature), [2002] 1 R.C.S. 249. Ruffo (Re), [2006] R.J.Q. 26 (C.A.). 
(2007) 38 R.D.U.S.

Les conflits

d'intérêts institutionnels

On trouve aussi dans la jurisprudence un exemple de conflit d'intérêts institutionnel relativement aux droits civiques des juges. Il concerne une disposition législative fédérale privant les juges de leur droit de vote aux élections fédérales. Au milieu des années 1980, la validité constitutionnelle de cette disposition est attaquée devant la Cour fédérale par deux membres de ce tribunal. Ces procédures ne sont pas contestées par le Procureur général du Canada. Elles sont donc accueillies par le juge suppléant désigné pour entendre le litige ${ }^{26}$, sans que ne soit abordée la question du conflit d'intérêts dans lequel celui-ci se trouve placé, puisque son jugement lui rend un droit de vote dont il était auparavant privé. Toutefois, deux aspects du jugement prennent une certaine importance à ce propos : d'une part, le jugement passe en revue différents arguments que le Procureur général du Canada aurait pu faire valoir pour soutenir la validité de la disposition contestée; d'autre part, le juge conclut ses motifs de jugement en déclarant que "la décision aurait fort bien pu favoriser l'une ou l'autre partie s'il y avait eu une véritable contestation" 27. Cette façon d'aborder le dossier visait de toute évidence à préserver l'apparence d'impartialité du tribunal, placé dans la position inusitée où l'absence d'un véritable débat entre les parties ne lui laissait guère d'autre choix que de donner raison aux juges ayant pris l'initiative du litige.

Par ailleurs, la jurisprudence contient plusieurs exemples de litiges ayant trait à la gestion des tribunaux et dans lesquels la doctrine de la nécessité a été invoquée. Ils concernent les ressources mises à la disposition des juges pour leur permettre d'exercer leurs fonctions, l'autorité du juge en chef au sein du tribunal, ou encore la validité des règles de pratique adoptées par l'ensemble des juges du tribunal.

Ainsi, en 1986, le juge en chef de la Cour supérieure intente devant le tribunal qu'il préside une action par laquelle il cherche à faire déclarer invalides diverses mesures gouvernementales, ayant

26. Muldoon c. Canada, [1988] 3 C.F. 628 (C.F. 1 1re inst.) [Muldoon].

27. Ibid. à la p. 636. 
pour objet de supprimer le poste de huissier-audiencier - un auxiliaire de justice présent dans une salle de cour pendant qu'un juge y siège - et de réduire le nombre de secrétaires mises à la disposition des juges du tribunal. D'entrée de jeu, le Procureur général du Québec demande la récusation du juge saisi d'une demande d'injonction provisoire visant à empêcher le gouvernement de mettre en œuvre des décisions déjà prises à cet égard : par le jugement qu'il est appelé à rendre, ce juge se trouve en effet à déterminer l'étendue de son propre droit de bénéficier des services d'un huissier-audiencier et d'une secrétaire.

Celui-ci motive de façon élaborée son refus de se récuser ${ }^{28}$. Il mentionne ainsi que "le requérant ne pouvait s'adresser à d'autres tribunaux, qu'à la Cour supérieure». Il fait aussi état de l'existence d'un recours alternatif permettant au gouvernement d'éviter, au sein de la Cour supérieure, le conflit d'intérêts causé par les procédures du juge en chef : la possibilité d'un renvoi, à la Cour d'appel, des questions en litige. Ayant expressément demandé à l'avocat représentant le Procureur général du Québec si le gouvernement avait l'intention de se prévaloir de cette procédure de renvoi, puis ayant constaté que le gouvernement n'avait pas manifesté d'intention en ce sens, le juge ajoute que, dans ces circonstances, "demander à tous et chacun des juges de la Cour supérieure de se récuser, équivaudrait à dénier au requérant son droit de s'adresser aux tribunaux'. Enfin, le juge prend appui sur la doctrine de la nécessité :

Troisièmement, le procureur du requérant invoque, alternativement, la doctrine de la nécessité. En effet, il peut arriver - comme dans le présent cas - qu'aucune cour ne soit habilitée à connaître d'une affaire si les principes

28. Gold c. Procureur général du Québec, [1986] R.J.Q. 2924 aux pp. 29262927 (C.S.). Quelques années auparavant, la Cour d'appel avait rejeté l'appel formé à l'encontre d'une ordonnance par laquelle un juge de la Cour supérieure avait contraint le directeur régional des services judiciaires et protonotaire de la Cour supérieure à mettre un huissier-audiencier à sa disposition chaque jour jusqu'à la fin de son terme: Shatilla c. Shatilla, [1982] C.A. 511. 
usuels de récusation sont appliqués. Les tribunaux ont alors développé la théorie de la nécessité. (...)

En conséquence, pour les motifs invoqués par le procureur du requérant, je suis d'avis de rejeter la demande en récusation, puisque le droit fondamental et constitutionnel d'être entendu par un arbitre judiciaire prime sur les inconvénients soulevés par le procureur des intimés.

Ce jugement marque une étape importante dans le traitement des conflits d'intérêts institutionnels au sein de la magistrature. En effet, la question y est abordée d'une manière quelque peu différente de celle auparavant utilisée par les tribunaux pour justifier l'application de la doctrine de la nécessité. Jusqu'à cette époque, la jurisprudence se plaçait surtout dans la perspective du tribunal saisi du litige pour traiter du conflit d'intérêts institutionnel : les motifs invoqués pour éviter la récusation insistent principalement sur la nécessité que le tribunal puisse exercer sa compétence. L'approche retenue dans cette affaire met plutôt l'accent sur la perspective du juge qui s'adresse au tribunal : il faut que celui-ci puisse faire valoir les droits dont il est titulaire et qu'il cherche à mettre en œuvre par ses procédures.

En ce qui concerne le mérite de la demande du juge en chef, la Cour supérieure ordonne le sursis des décisions du gouvernement qui supprimaient des postes d'huissiers-audienciers et qui réduisaient le ratio des secrétaires de juges de la Cour supérieure à une secrétaire pour deux juges. Une demande de permission d'en appeler de la partie du jugement relative à la demande de récusation est accordée par la Cour d'appel, mais l'appel n'aura pas de suite 29 .

29. Procureur général du Guébec c. Gold, (2 juin 1986), Montréal 500-46000199-861, J.E. 86-612 (C.A.) [Gold]. Vingt ans plus tard, un autre juge de la Cour supérieure considère que cet appel, théoriquement toujours pendant, a été déserté : Société Radio-Canada c. Procureur général du Québec, [2006] R.J.Q. 2826 à la p. 2831 (C.S.). 
Quelques années plus tard, les juges en chef de tous les tribunaux judiciaires du Québec intentent devant la Cour supérieure une action visant à faire déclarer invalides diverses mesures gouvernementales concernant l'utilisation des stationnements réservés aux juges de la Cour d'appel, de la Cour supérieure et de la Cour du Québec dans les palais de justice de la province. Sans aborder la question de son conflit d'intérêts, le juge saisi d'une demande de sursis accessoire à ces procédures prononce, dans le jugement par lequel il donne raison aux juges en chef, des conclusions explicites relativement au processus qui aurait pu conduire à sa récusation :

Déclare que cette Cour a compétence pour entendre et disposer de la présente requête pour émission d'une injonction interlocutoire provisoire et pour l'émission d'une ordonnance de sursis.

Déclare les articles 234 et 242 du Code de procédure civile inapplicables et inopérants dans la mesure où ils empêchent les requérants de faire valoir les droits et privilèges constitutionnels des juges de la Cour d'appel, Cour supérieure et Cour du Québec.

Réserve aux intimés le droit d'invoquer l'application de ces dispositions, si bon leur semble, plus tard. 30

Une étape additionnelle est donc franchie à cette occasion. Le jugement reprend la perspective utilisée par la Cour supérieure quelques années plus tôt et met l'emphase sur la possibilité, pour les juges ayant pris l'initiative du litige, de pouvoir faire valoir leurs droits devant le tribunal. Il en tire toutefois une conséquence inédite : ce jugement est le premier - et le seul - qui déclare inopérantes, dans un tel contexte, les dispositions législatives exigeant la récusation d'un juge en cas de conflit d'intérêts. Dans aucune partie du jugement on ne trouve de motifs expliquant cette conclusion, ni même une identification des

30. Bisson c. Procureur général du Guébec, [1992] R.J.Q. 1947 à la p. 1959 (C.S.). 
principes constitutionnels permettant au tribunal de déclarer ces dispositions législatives inopérantes. La position du tribunal n'en est pas moins claire : les droits et privilèges constitutionnels de la magistrature doivent pouvoir être exercés et l'obligation de récusation ne peut y faire échec. Cette prise de position explique peutêtre qu'aucune demande de récusation n'ait été subséquemment formulée à l'égard du juge saisi du mérite du litige, qui donne lui aussi raison aux juges en chef ${ }^{31}$.

Divers litiges relatifs à l'autorité du juge en chef au sein du tribunal qu'il préside ont surgi au cours des dernières années au sein de certains tribunaux composés de juges de nomination provinciale, que ce soit en rapport avec l'assignation de fonctions judiciaires à des juges ${ }^{32}$, à propos du contrôle des sommes utilisées pour le perfectionnement professionnel ${ }^{33}$, ou relativement aux pouvoirs du juge en chef en matière déontologique ${ }^{34}$. Ces affaires ne présentaient cependant aucun véritable conflit d'intérêts institutionnel, d'une part parce que ce sont les tribunaux supérieurs de la province qui en étaient saisis, et non les tribunaux dont faisaient partie les juges en litige les uns avec les autres, et d'autre part en raison de l'encadrement législatif spécifique à chacun de ces divers tribunaux, qui réduit la portée des jugements qui découlent de tels litiges à l'égard des autres membres de la magistrature.

31. Bisson c. Procureur général du Québec, [1993] R.J.Q. 2581 (C.S.).

32. Au Québec, voir : Ruffo c. St-Louis, (26 mars 1999), Québec 200-05009095-980, REJB 1999-11796 (C.S.); Ruffo c. St-Louis, (7 avril 2000), Québec 200-09-002563-994, REJB 2000-17702 (C.A.); Ruffo c. St-Louis, [2004] R.J.Q. 137 (C.S.). En Alberta, voir : Reilly v. Wachowich, (1999) 229 A.R. 218 (Q.B. Alb.), conf. par (1999) 228 A.R. 303 (C.A. Alb.); Reilly v. Provincial Court of Alberta Chief Judge, (2001) 192 D.L.R. (4th) 540 (C.A. Alb.). Au Nouveau-Brunswick, voir : New Brunswick (Chief Judge of the Provincial Court) v. Mackin, (1996) 134 D.L.R. (4th) 243 (C.A.N.-B.), autorisation de pourvoi à la C.S.C. refusée, [1996] 3 R.C.S. x.

33. Alberta (Provincial Court Judge) v. Alberta (Provincial Court Chief Judge), [2006] 9 W.W.R. 291 (Q.B. Alb.).

34. Ruffo c. Gobeil, [1989] R.J.Q. 1943 (C.S.). 
Il en est cependant autrement d'une affaire reliée à la conduite du juge en chef de la Cour fédérale du Canada, survenue au milieu des années 1990. À cette époque, la structure de la Cour fédérale était la suivante : la Cour était séparée en une division de première instance et une division d'appel; le juge en chef présidait l'ensemble de la Cour, alors que le juge en chef adjoint présidait la division de première instance. Dans le cadre d'un dossier dont le juge en chef adjoint est saisi, en première instance, diverses communications interviennent entre le juge en chef de la Cour et un représentant du gouvernement fédéral, partie au litige. Le juge en chef fait part au juge en chef adjoint des préoccupations du gouvernement fédéral à propos des délais mis par le tribunal à traiter le dossier et le juge en chef adjoint s'engage à s'occuper du dossier avec diligence.

Saisie d'une demande de suspension des procédures par la partie tenue à l'écart de ces communications, la division de première instance de la Cour doit déterminer les conséquences juridiques de la conduite du juge en chef à l'égard du dossier dont le juge en chef adjoint est saisi. Pour ce faire, elle doit apprécier tant la conduite du juge en chef adjoint, par rapport aux délais mis à traiter le dossier, que celle du juge en chef de la Cour fédérale, en ce qui a trait à son intervention dans un dossier dont il n'est pas saisi. Cette question place donc dans une situation délicate le juge saisi de la demande de suspension, et ce, par rapport à chacun de ses deux juges en chef. Il conclut que l'ingérence du juge en chef de la Cour dans le dossier dont le juge en chef adjoint était saisi a fait perdre leur indépendance à l'ensemble des juges de la division de première instance et, en conséquence, ordonne la suspension des procédures ${ }^{35}$.

Ce jugement est porté en appel devant la Cour d'appel fédérale. Tout comme le juge de première instance, mais d'une manière différente qui découle de leur propre position dans la hiérarchie de la cour, les juges de la Cour d'appel fédérale sont

35. Canada (Ministre de la Citoyenneté et de l'Immigration) c. Tobiass, [1996] 2 C.F. 729 (C.F. $1^{\text {re }}$ inst.). 
aussi placés dans une situation délicate. De façon préliminaire, la Cour d'appel fédérale est appelée à décider si ses membres possèdent l'impartialité requise pour apprécier la conduite de leur juge en chef. Dans la décision qu'elle rend à ce propos, elle conclut qu'elle ne peut refuser d'exercer la compétence exclusive qui lui est attribuée par la loi à l'égard du jugement de première instance. Il existe cependant une divergence quant aux motifs utilisés par les juges pour parvenir à cette conclusion. L'un d'entre eux considère que la doctrine de la nécessité doit recevoir application :

It is a widely accepted and fundamental rule in the administration of justice that where a judge is interested in the result of litigation he or she cannot sit. This rule is subject to the operation of the common law doctrine of necessity (...).

As the position of the respondents is that all members of the Court are affected by the alleged bias and so disqualified from sitting, it is not possible to constitute another panel. ${ }^{36}$

Un autre juge considère inutile le recours à cette doctrine, considérant qu'une personne raisonnable ne pourrait conclure que le besoin d'analyser la conduite du juge en chef rend inhabiles les juges saisis de l'appel, qui se trouvent être les trois plus anciens membres de la Cour ${ }^{37}$. Au mérite de l'appel, la Cour d'appel fédérale casse le jugement de première instance ${ }^{38}$, non sans que l'un des juges ne souligne la difficulté de conserver une nécessaire distance par rapport au dossier :

Il convient de prendre acte dès le départ du caractère particulièrement difficile de cette affaire étant donné son sujet et les circonstances inusitées qui en ont accompagné le déroulement devant la Cour. [...] Et enfin, le fait que les

36. Canada (Minister of Citizenship and Immigration) c. Tobiass, (1997) 208 N.R. 49 au para. 19 (C.A.F.).

37. Ibid. aux para. 12-14.

38. Canada (Ministre de la Citoyenneté et de l'Immigration) c. Tobiass, [1997] 1 C.F. 828 (C.A.F.). 
agissements du juge en chef de cette Cour aient été mis en cause oblige à un surcroît d'efforts pour ne pas se voir personnellement engagé dans le débat. 39

La position embarrassante des juges de la Cour fédérale n'est pas commentée par la Cour suprême du Canada qui, en appel de l'arrêt de la Cour d'appel fédérale, considère que la suspension d'instance n'était pas le remède approprié, étant donné que les juges de la division de première instance autres que le juge en chef adjoint pouvaient décider du litige en toute indépendance ${ }^{40}$.

La doctrine de la nécessité a aussi été mentionnée dans deux décisions de la Cour supérieure analysant la validité de certaines règles de pratique. Dans un cas, le tribunal était saisi d'une requête visant à faire déclarer nulles les dispositions des règles de pratique de la Cour supérieure et de celles de la Cour du Québec exigeant le port de la toge par les avocats. Considéré comme sérieux par l'une des parties, le problème résultant du fait que le tribunal était appelé à statuer sur la validité de ses propres règlements est résolu par la Cour supérieure par l'application de la doctrine de nécessité ${ }^{41}$. Dans l'autre cas, la Cour supérieure était saisie d'une demande visant à faire déclarer nulle une disposition de ses règles de pratique limitant la présence des caméras et la prise d'entrevues à des endroits spécifiques dans les palais de justice. N'ayant pas participé au processus ayant conduit à l'adoption de cette règle, le juge saisi du dossier considère qu'il n'a pas besoin de recourir à la doctrine de la nécessité pour justifier

39. Ibid. à la p. 840.

40. Canada (Ministre de la Citoyenneté et de l'Immigration) c. Tobiass, [1997] 3 R.C.S. 391. Les juges de la Cour suprême du Canada n'étaient évidemment pas en conflit d'intérêts dans ce litige, qui concernait le fonctionnement interne d'un tribunal hiérarchiquement inférieur.

41. Dury c. Procureur général du Buébec, (4 juin 2002), Montréal 500-05070985-021, J.E. 2002-1396, au para. 4 (C.S.). La requête est rejetée par le tribunal. 
sa compétence à trancher le litige, d'autant plus que les parties avaient consenti à ce qu'il se saisisse du dossier ${ }^{42}$.

\section{LA DOCTRINE DE LA NÉCESSITÉ}

Principe d'origine jurisprudentielle, la doctrine de la nécessité n'a reçu qu'une attention limitée de la part des auteurs ${ }^{43}$. Ses fondements n'ont été identifiés que de façon sommaire et ses modalités d'application restent encore largement indéfinies.

\subsection{Les fondements de la doctrine}

L'énoncé le plus complet de la doctrine de la nécessité en droit canadien se trouve dans un arrêt rendu par la Cour suprême du Canada dans le cadre d'une série de litiges concernant la rémunération des juges de nomination provinciale de certaines provinces $^{44}$. Ayant décidé dans un arrêt précédent que le mode de fixation de leur rémunération ne respectait pas les exigences constitutionnelles, la Cour était alors appelée à déterminer la validité des jugements rendus par ces juges avant que ne soient mis en place le mécanisme constitutionnellement requis pour établir leur rémunération. Le problème soulevé dans ce litige avait donc trait à la valeur des jugements rendus par des tribunaux ne présentant

42. Société Radio-Canada c. Procureur général du Québec, [2006] R.J.Q. 2826 à la p. 2831 (C.S.). La contestation est rejetée, mais ce jugement a fait l'objet d'un appel.

43. Les auteurs se contentent le plus souvent de mentionner l'existence de la doctrine de la nécessité, sans autre analyse; à titre d'exemples : Shimon Shetreet, Judges onTrial - A Study of the Appointment and Accountability of the English Judiciary, Amsterdam-New York-Oxford, North Holland Publishing Company, 1976 à la p. 304. John O. Wilson, A Book for Judges, supra note 3 à la p. 29. Denis Ferland et Benoît Emery, Précis de procédure civile du Québec, vol. 1, 4e éd., Cowansville, Yvon Blais, 2003 à la p. 388. Louis-Paul Cullen, "La récusation d'un juge saisi d'un litige civil", dans Service de la formation permanente du Barreau du Québec, Développements récents en déontologie, droit professionnel et disciplinaire, Cowansville, Yvon Blais, 2001, 215 à la p. 239; Bryden, supra note 3 aux pp. 579580.

44. Renvoi relatif à la rémunération des juges de la Cour provinciale (Î-P-É), supra note 16. 
pas les garanties nécessaires d'indépendance et la Cour suprême du Canada y applique la doctrine de la nécessité non pour résoudre des situations de conflits d'intérêts au sein de la magistrature, mais pour pallier un manque d'indépendance judiciaire.

Les sources utilisées par la Cour suprême du Canada pour servir d'assise à ce principe sont pour la plupart étrangères au droit canadien. Elles ne traitent d'ailleurs pas toutes de conflits d'intérêts institutionnels : c'est notamment le cas d'un arrêt de la House of Lords ${ }^{45}$ fréquemment cité par les tribunaux canadiens à titre d'autorité en cette matière, où la fonction exercée par le juge placé en situation de conflit d'intérêts se limitait à une simple formalité administrative ${ }^{46}$. Néanmoins, la définition que la Cour suprême du Canada donne de la doctrine de la nécessité à cette occasion est suffisamment générale pour englober les situations de conflit d'intérêts :

La règle générale selon laquelle un juge qui n'est pas impartial est inhabile à entendre une affaire connaît une exception. Cette exception permet à un juge qui, sans elle, serait inhabile à connaître d'un litige d'entendre quand même cette affaire dans les cas où il n'y a pas de juge impartial en mesure de le remplacer. En effet, le droit reconnaît que, dans certaines situations, il est préférable

45. Dimes $v$. The Proprietors of the Grand Junction Canal, [1852] 3 H.L. Cas. 759. Pour pouvoir être portée en appel auprès de la House of Lords, une ordonnance d'injonction rendue par la Court of Chancery devait être enregistrée par le Chancelier, son président, qui était actionnaire de la compagnie ayant demandé l'émission de l'ordonnance d'injonction. C'est dans ce contexte que le tribunal invoque la doctrine de la nécessité pour justifier l'enregistrement de la décision par le Chancelier, seul membre de la cour apte à procéder à cet enregistrement (p. 787). Contrairement aux situations de conflit d'intérêts institutionnel, ce n'est donc pas en sa qualité de juge, mais à titre purement personnel, que le Chancelier était intéressé au litige.

46. "It did not involve his making any decision on the merits of the case." : R.R.S. Tracey, supra note 7 à la p. 630. 
d'avoir un juge qui n'est pas indépendant ni impartial plutôt que de ne pas avoir de juge du tout. ${ }^{47}$

Cette définition est semblable à celle donnée par la doctrine canadienne et britannique. Le Conseil canadien de la magistrature présente cette doctrine de la façon suivante:

En vertu du principe de la nécessité, les juges qui devraient autrement se récuser peuvent entendre et décider une instance si l'omission de procéder risque d'entraîner une injustice. Tel pourrait être le cas si la remise ou l'avortement du procès causait des difficultés excessives ou si aucun autre juge n'était raisonnablement disponible qui ne serait pas lui-même inhabile à siéger. ${ }^{48}$

L'un des rares auteurs ayant procédé à l'étude de cette doctrine en droit anglais la formule d'une manière similaire :

In the field of public law, the doctrine of necessity is invoked to allow an otherwise disqualified adjudicator to hear and dispose of a case when no other qualified person is available. The underlying principle has been said to be the prevention of "a failure of justice». 49

Aux termes de ces définitions, il appert que la présence cumulative de trois conditions est requise pour permettre l'application de la doctrine de la nécessité par un tribunal. En premier lieu, il doit exister un défaut irrémédiable concernant l'impartialité ou l'apparence d'impartialité du juge saisi du litige. Cette condition est présente dans les situations de conflit d'intérêts institutionnel puisqu'un juge a nécessairement intérêt dans un litige qui concerne le statut de la magistrature dont il fait partie. En second lieu, aucun autre juge présentant toutes les

47. Renvoi relatif à la rémunération des juges de la Cour provinciale (Î-P-É), supra note 16, para. 4.

48. Conseil canadien de la magistrature, supra note 3 à la p. 47, para. E.17; voir aussi p. 29, para. E.3.

49. Tracey, supra note 7 à la p. 628. 
garanties d'impartialité, et qui pourrait être saisi du litige en temps opportun, ne doit être disponible. Cette condition est aussi présente dans les situations de conflit d'intérêts institutionnel, puisque tous les juges d'un même tribunal ont un intérêt semblable dans le litige. En troisième lieu, il ne doit exister aucun autre forum permettant de déterminer de manière appropriée les droits et les obligations des parties au litige. Cette condition est remplie lorsque le tribunal saisi du litige possède une compétence exclusive pour en décider. Il en résulte que l'application de la doctrine de la nécessité dans un contexte de conflit d'intérêts institutionnel est entièrement justifiée.

De toute évidence, la doctrine de la nécessité constitue un exercice de conciliation de deux valeurs fondamentales, mises en cause par les situations de conflits d'intérêts: d'une part, l'impartialité dont le tribunal doit faire preuve lorsqu'il entend et tranche des litiges; d'autre part, la primauté du droit, qui garantit à toute personne la possibilité de s'adresser à un tribunal pour faire valoir les droits dont elle est titulaire. Élaborée dans un cadre constitutionnel bien différent de celui que connaît aujourd'hui le Canada, la doctrine de la nécessité doit maintenant être façonnée en fonction du statut qu'occupent ces valeurs dans le système juridique canadien.

L'exigence d'impartialité représente à n'en pas douter la caractéristique la plus éminente des institutions judiciaires. Spécifiquement mentionnée à l'article $11 \mathrm{~d}$ ) de la Charte canadienne des droits et libertés ${ }^{50}$, l'impartialité judiciaire fait aussi partie des principes de justice fondamentale protégés par l'article 7 de la Charte $^{51}$, ainsi que des principes de justice naturelle ${ }^{52}$. À ce titre,

50. Partie I de la Loi constitutionnelle de 1982, constituant l'annexe B de la Loi de 1982 sur le Canada (R.-U.), 1982, c. 11. L'article $11 \mathrm{~d})$ prévoit que "Tout inculpé a le droit [...] d'être présumé innocent tant qu'il n'est pas déclaré coupable, conformément à la loi, par un tribunal indépendant et impartial à l'issue d'un procès public et équitable".

51. Ibid. L'article 7 prévoit que "Chacun a le droit à la vie, à la liberté et à la sécurité de sa personne; il ne peut être porté atteinte à ce droit qu'en conformité avec les principes de justice fondamentale". 
elle représente "l'une des obligations fondamentales des tribunaux de l'ordre judiciaire» 53 et constitue le pivot des principes déontologiques qui encadrent la conduite des membres de la magistrature $^{54}$. Les cours de justice sont ainsi appelées à respecter "les plus hautes normes d'impartialité»55. Un excès de compétence sanctionne le manquement du tribunal à cette obligation, quel que soit par ailleurs le mérite du jugement qui est rendu ${ }^{56}$.

Depuis l'adoption de la Loi constitutionnelle de 1982, la Cour suprême du Canada a porté une attention particulière au contenu de cette notion. Elle a défini l'obligation d'impartialité comme signifiant que l'état d'esprit et l'attitude du juge relativement aux parties à un litige et aux questions qui y sont soulevées doivent être exempts de préjugés, réels ou apparents ${ }^{57}$. L'impartialité vise ainsi à maintenir la confiance des justiciables dans leurs institutions judiciaires 58 et à faire en sorte que les jugements soient rendus conformément aux règles de droit, sur la base de la preuve entendue par le tribunal59. Un juge doit aborder

52. Pearlman c. Comité judiciaire de la Société du Barreau du Manitoba, [1991] 2 R.C.S. 869 aux pp. 882-883; Ruffo c. Conseil de la magistrature, [1995] 4 R.C.S. 267 à la p. 296.

53. Cie pétrolière impériale ltée c. Québec (ministre de l'Environnement), [2003] 2 R.C.S. 624 au para. 22.

54. Les principes déontologiques développés par le Conseil canadien de la magistrature à l'attention des juges de nomination fédérale traitent longuement de l'obligation d'impartialité : Conseil canadien de la magistrature, supra note 3 aux pp. 27-50. En ce qui concerne les juges de nomination provinciale au Québec, deux articles du Code de déontologie de la magistrature, R.R.Q. c. T-16 r. 4.1, traitent de ce sujet: l'article 5 énonce que le juge doit de façon manifeste être impartial et objectif et l'article 4 lui impose l'obligation de prévenir tout conflit d'intérêts.

55. R. c. S. (R.D.), [1997] 3 R.C.S. 484 aux para. 93, 118.

56. Ibid. à la p. 526.

57. Valente c. La Reine, [1985] 2 R.C.S. 673 à la p. 685; "L'équité et l'impartialité doivent être à la fois subjectivement présentes et objectivement démontrées dans l'esprit de l'observateur renseigné et raisonnable" : R. c. S. (R.D.), supra note 55 à la p. 524.

58. Bande indienne Wewaykum c. Canada, [2003] 2 R.C.S. 259 à la p. 287-288 [Wewaykum].

59. R. c. Généreux, [1992] 1 R.C.S. 259 à la p. 282. 
les litiges avec un esprit ouvert60, demeurer désintéressé par rapport au résultat et être susceptible d'être persuadé par la preuve et les arguments qui lui sont soumis ${ }^{61}$. L'exigence d'impartialité lui impose de trancher les litiges qui lui sont soumis "en toute liberté d'esprit, sans être influencé par des intérêts personnels et des pressions externes" 62 .

L'une des conséquences du principe d'impartialité est qu'une personne ne peut agir comme juge dans sa propre cause ou décider d'un litige dans lequel elle a un intérêt personnel. Il existe un risque de conflit d'intérêts, selon le Conseil canadien de la magistrature, "lorsque l'intérêt personnel du juge lou de ses proches) s'oppose à son devoir de rendre la justice avec impartialité»63. La House of Lords résume ainsi cette règle capitale :

One of the cornerstones of our legal system is the impartiality of the tribunals by which justice is administered. In civil litigation the guiding principle is that no one may be a judge in his own cause : nemo debet esse judex in propria causa. It is a principle which is applied much more widely than a literal interpretation of the words might suggest. It is not confined to cases where the judge is a party to the proceedings. It is applied also to cases where he has a personal or pecuniary interest in the outcome, however small. 64

Les situations de conflits d'intérêts institutionnels placent les membres de la magistrature en contradiction manifeste avec l'exigence d'impartialité. La position délicate dans laquelle est placé le juge saisi d'un litige relié aux modalités de la fonction judiciaire est évidente. Elle a été décrite comme suit par l'un d'eux, au tournant des années 1980, à une époque où la doctrine de la

60. Bande indienne Wewaykum, supra note 58 à la p. 288.

61. R. c. S. (R.D.), supra note 55 à la p. 528.

62. Cie pétrolière impériale ltée c. Québec (ministre de l'Environnement), [2003] 2 R.C.S. 624 au para. 28.

63. Conseil canadien de la magistrature, supra note 3 à la p. 42, au para. E.2.

64. R. v. Bow Street Metropolitan Stipendiary Magistrates, Ex parte Pinochet Ugarte (No. 2), [1999] 2 W.L.R. 272 à la p. 288 (H.L.). 
nécessité n'avait encore fait l'objet que d'une seule application en droit canadien dans un contexte de conflit d'intérêts institutionnel :

Avant de procéder à l'examen de ces questions, il m'incombe de déclarer l'intérêt que j'ai dans l'issue du litige. Lorsque, comme en l'espèce, un juge ayant un intérêt personnel dans l'issue du litige est néanmoins obligé d'instruire l'affaire parce qu'aucun autre juge désintéressé et ayant la compétence voulue n'est disponible, la tâche devient deux fois plus lourde. À cause de son intérêt personnel dans l'issue du litige, le juge, pour redoubler de prudence et par souci réel que justice soit faite en toute impartialité, est obligé de se prémunir contre le danger de pencher indûment en faveur du point de vue opposé à cet intérêt ainsi que contre le danger d'être inconsciemment influencé par celui-ci. C'est là un numéro d'équilibriste qui demande des acrobaties de jugement et d'introspection morale, talent que bien peu de personnes possèdent et qu'encore moins sont appelées à exercer. Enfin, aussi consciencieusement que la tâche soit accomplie, il reste toujours la possibilité réelle qu'aux yeux du citoyen ordinaire, cela semblera être rien de moins que de l'inceste judiciaire. ${ }^{65}$

Une décennie plus tard, alors que le nombre de litiges relatifs au statut de la magistrature a déjà commencé à augmenter, un juge de la Cour supérieure exprime encore des préoccupations similaires :

Enfin, les défendeurs n'ont pas soulevé l'application des articles 234 à 242 du Code de procédure civile concernant la récusation. Cependant, je réalise que j'ai une tâche bien délicate et que pour l'accomplir je dois mettre de côté ma propre conception des événements pour ne m'en tenir qu'aux faits qui m’ont été prouvés. 66

65. Beauregard, supra note 12 aux pp. 549-550.

66. Bisson c. Procureur général du Québec, [1993] R.J.Q. 2581 à la p. 2586 (C.S.). 
Que le conflit d'intérêts existe parce que le juge bénéficie directement des conclusions que renferme son jugement, ou parce que les principes de droit dont il s'inspire encadrent son propre statut de juge, il lui manque la distance et le détachement nécessaires pour lui permettre, à tout le moins en apparence, de parvenir à cette entière impartialité qui commande le respect des justiciables. De plus, même en maintenant la plus stricte indépendance, il doit être particulièrement difficile pour un juge de faire complètement abstraction des effets que son jugement peut produire à l'égard de ses collègues et de risquer ainsi leur réprobation. Cet élément est caractéristique aux conflits d'intérêts institutionnels: le jugement produit ses effets au sein même de la collectivité à laquelle est socialement rattaché le juge qui le prononce.

La primauté du droit est consacrée par la Charte canadienne des droits et libertés comme un principe fondamental de la société canadienne ${ }^{67}$. Le statut constitutionnel de ce principe, qui s'impose au pouvoir judiciaire68, apparaît donc incontestable 69 . Diversement apprécié par les auteurs ${ }^{70}$, sa signification n'a pas encore été précisément fixée par la jurisprudence ${ }^{71}$. On peut

67. Le préambule de la Charte, supra note 50, énonce ainsi que "le Canada est fondé sur des principes qui reconnaissent la suprématie de Dieu et la primauté du droits.

68. Colombie-britanique c. Imperial Tobacco Canada Ltée, [2005] 2 R.C.S. 473 à la p. 498.

69. Renvoi : Droits linguistiques au Manitoba, [1985] 1 R.C.S. 721 à la p. 750 .

70. Les auteurs donnent au principe de la primauté du droit une portée très variable. À titre d'exemple des différentes conceptions qui ont été proposées pour en déterminer le contenu, voir : Allan C. Hutchinson et Patrick Monahan, The Rule of Law - Ideal or Ideology, Toronto, Carswell, 1987; Daniel Mockle, "L'état de droit et la théorie de la rule of law", (1994) 35 C. de D. 823; Luc B. Tremblay, "La théorie constitutionnelle canadienne et la primauté du droit", (1994) 39 R.D. McGill 101; Henri Brun et Guy Tremblay, Droit constitutionnel, 4e éd., Cowansville, Éditions Yvon Blais, 2002 à la p. 679 et s.; Warren J. Newman, "The Principles of the Rule of Law and Parliamentary Sovereignty in Constitutional Theory and Litigation", (20042005) 16 R.N.D.C. 175 ;Peter W. Hogg et Cara F. Zwibel, "The rule of law in the Supreme Court of Canada", (2005) 55 Univ. of Toronto. L. J. 715.

71. L'arrêt de principe de la Cour suprême du Canada à propos de la primauté du droit ne contient qu'une description assez générale de son contenu: 
considérer la primauté du droit comme le principe fondateur du droit, celui qui sert à implanter le système juridique dans la société et à assurer sa prépondérance. Ce principe sert donc d'assise commune à l'ensemble des règles de droit. Par voie de conséquence, il fonde l'autorité des divers mécanismes destinés à établir les règles de droit, à les mettre en ouvre et à les faire respecter par les justiciables.

Le principe de la primauté du droit n'a fait l'objet que de quelques applications concrètes dans la jurisprudence ${ }^{72}$. L'une d'elles consiste à faire de ce principe le fondement du droit des justiciables de s'adresser à un tribunal pour faire valoir leurs droits. Initialement invoqué pour empêcher toute entrave à l'accès aux lieux où siègent les cours de justice ${ }^{73}$, le principe de la primauté du droit est aussi utilisé pour garantir aux justiciables la possibilité de saisir un tribunal afin d'exercer un recours ${ }^{74}$. C'est précisément ce volet de la primauté du droit qui est affecté par les règles qui forcent la récusation des membres d'un tribunal, lorsque de telles règles ont pour conséquence de rendre inhabiles l'ensemble des juges qui possèdent la compétence de trancher le litige.

En tant que sujet de droit, le juge est titulaire de certains droits et il est aussi redevable de certaines obligations, qui trouvent leur source dans divers principes constitutionnels, dans des dispositions législatives et dans la common law. Il est donc néces-

Renvoi : Droits linguistiques au Manitoba, [1985] 1 R.C.S. 721 aux pp. 747752.

72. À ce sujet, voir : Luc Huppé, "La concrétisation de la primauté du droit dans l'ordre juridique", (1997) 38 C. de D. 805.

73. B.C.G.E.U. c. Colombie-Britannique (Procureur général), [1988] 2 R.C.S. 214 à la p. 230.

74. Fraternité des préposés à l'entretien des voies - Fédération du réseau Canadien Pacifique c. Canadien Pacifique Ltée, [1996] 2 R.C.S. 495 aux pp. 501502; à ce sujet, voir aussi : Société de développement de la Baie James c. Compagnie de construction et de développement cris ltée, [2001] R.J.Q. 1726 à la p. 1730 (C.A.); Leroux c. La Reine, (19 février 2007), Montréal 500-10-003550-066, J.E. 2007-507 au para. 14 (C.A.); ColombieBritannique (Procureur général) c. Christie, 2007 CSC 21 au para. 16. 
saire que les personnes concernées par ces droits et ces obligations - et au premier chef les juges eux-mêmes - disposent de la possibilité de s'adresser à un tribunal pour exercer, en cas de conflit, les recours qui y sont afférents. Les règles de récusation ne peuvent, sans contrevenir au principe de la primauté du droit, faire échec à cette faculté et priver les juges ou leurs opposants de l'accès à un tribunal. La possibilité de saisir un tribunal pour faire valoir ses droits constitue une composante tellement essentielle au système juridique que la validité des mesures susceptibles d'y faire échec pourrait être attaquée ${ }^{75}$. Quels que soient les objectifs qui inspirent de telles mesures, et même lorsqu'elles sont fondées sur un principe aussi important que la préservation de l'impartialité judiciaire, elles ne peuvent avoir pour conséquence d'écarter totalement l'accès à un tribunal.

La doctrine de la nécessité trouve donc son fondement dans le principe de la primauté du droit76. Dans le contexte des conflits d'intérêts institutionnels, elle vise à garantir aux juges et aux autres justiciables affectés par des différends concernant la fonction judiciaire l'existence d'un recours devant un tribunal pour y mettre fin ${ }^{77}$. Cette doctrine apparaît en outre indispensable pour pré-

75. Bien que le tribunal n'ait pas exprimé de motifs en ce sens, on peut expliquer de cette façon la conclusion prononcée par la Cour supérieure dans Bisson c. Procureur général du Québec, [1992] R.J.Q. 1947 à la p. 1959 (C.S.), déclarant inopérants les articles 234 et 242 du Code de procédure civile dans la mesure où ils empêchent les juges de faire valoir leurs droits et leurs privilèges constitutionnels.

76. C'est aussi l'idée exprimée sans autres détails par la Cour suprême du Canada : Renvoi relatif à la rémunération des juges de la Cour provinciale $(\hat{I}-$ $P$-É), supra note 16 au para. 6.

77. Le droit américain reconnaît pareillement que le but de la doctrine de la nécessité est de réaliser le droit des justiciables à un forum : Debra Lyn Bassett, "Recusal and the Supreme Court", (2004-2005) 56 Hastings L. J. 657 à la p. 682; S.E. BLOOM, Judicial Bias and Financial Interest as Grounds for Disqualification of Federal Judges, (1984-1985) 35 Case Western Reserve Law Review 662 aux pp. 679-680; John Leubsdorf, "Theories of Judging and Judge Disqualification", (1987) 62 N.Y.U.L. Rev. 237 à la p. 241. La décision de principe relative à la doctrine de la nécessité en droit américain émane de la Cour suprême des Etats-Unis. Elle découle d'un litige dans lequel divers juges de cours de district avaient intenté, devant une cour de district, une action visant à faire invalider des dispositions lé- 
server l'égalité entre les justiciables: tout comme leurs concitoyens, les juges doivent être assujettis à des règles de droit dont la sanction relève des institutions judiciaires de l'État. La volonté d'assurer une justice impartiale ne peut être portée au point où une partie des justiciables pourraient être privés de la possibilité de s'adresser à des instances judiciaires pour faire trancher les litiges qui les touchent. Il serait paradoxal que ceux-là même qui exercent des fonctions judiciaires soient, lorsque surviennent des litiges relatifs à la charge qu'ils occupent, exclus du bénéfice que procure à la société l'existence de tribunaux.

\subsection{Les modalités de la doctrine}

Les principes juridiques destinés à maintenir l'impartialité judiciaire doivent donc faire l'objet de certains aménagements, dans la mesure requise pour permettre la réalisation effective de la primauté du droit. Telle est la raison d'être de la doctrine de la nécessité. Toutefois, cette doctrine doit elle-même être assortie de certaines modalités, lorsqu'elle est invoquée dans le cadre d'un conflit d'intérêts institutionnel, afin de porter le moins possible atteinte à l'impartialité judiciaire. En effet, étant donné le statut constitutionnel de l'obligation d'impartialité, la doctrine de la nécessité ne peut trouver de légitimité qu'en minimisant les entorses qu'elle y apporte. Bien qu'elle soit justifiée par le principe de la primauté du droit enchâssé dans le préambule de la Charte canadienne des droits et libertés, cette règle de common law doit néanmoins demeurer compatible avec l'ensemble des dispositions de la Constitution canadienne.

Ce volet est pour l'instant peu développé. Deux limites très générales ont été posées par la Cour suprême du Canada à l'application de la doctrine de la nécessité78 : elle ne s'applique pas dans les cas où elle entraînerait une injustice substantielle et elle ne doit être appliquée que dans la mesure justifiée par la nécessi-

gislatives touchant leur rémunération : United States v. Will, 449 U.S. 200 (1980).

78. Renvoi relatif à la rémunération des juges de la Cour provinciale (Î-P-É), supra note 16 au para. 7 . 
té. De façon concrète, des règles plus précises peuvent sans doute être formulées; les divers jugements qui ont eu recours à cette doctrine dans le contexte spécifique des conflits d'intérêts institutionnels fournissent d'ailleurs certaines pistes de réflexion à ce sujet.

On peut poser comme première modalité que le juge qui considère la possibilité d'invoquer la doctrine afin de demeurer saisi d'un litige doit d'abord envisager les autres voies de recours qui s'offrent aux parties. La protection offerte par la primauté du droit ne garantit pas l'accès à un tribunal spécifique, non plus qu'elle n'accorde aux parties le choix absolu du tribunal, s'il en existe plusieurs compétents. Elle vise plutôt à assurer aux justiciables le bénéfice d'une instance judiciaire pour trancher les litiges qui les concernent.

Le Canada possède une structure judiciaire composée de nombreuses instances, de sorte que si le litige qui suscite un conflit d'intérêts institutionnel entre également dans la compétence d'un tribunal présentant une meilleure garantie d'impartialité que celui qui en est saisi, il devrait lui être référé. C'est vraisemblablement dans cet esprit qu'un juge de la Cour supérieure s'est informé de l'intention du gouvernement du Guébec, partie aux procédures dont il était saisi, quant à la possibilité de procéder à un renvoi auprès de la Cour d'appel relativement aux questions faisant l'objet du litige, qui ne touchaient que les juges de la Cour supérieure ${ }^{79}$. Pour le tribunal saisi du litige, il ne serait toutefois approprié de décliner sa compétence et de renvoyer le dossier à un tribunal possédant une compétence concurrente à la sienne que dans la mesure où les juges de cet autre tribunal ne sont pas eux-mêmes placés dans une situation de conflit d'intérêts ou encore si le conflit d'intérêts s'y manifeste de façon moins prononcée.

Si le litige relève de la compétence exclusive du tribunal qui en est saisi, mais qu'il s'agit d'un tribunal hiérarchiquement infé-

79. Gold c. Procureur général du Québec, [1986] R.J.Q. 2924 (C.S.). 
rieur, assujetti au pouvoir de contrôle et de surveillance, il pourrait sembler préférable que le litige soit renvoyé au tribunal de droit commun. En effet, en raison du statut constitutionnel de l'obligation d'impartialité, la validité des dispositions législatives qui attribuent une compétence exclusive à un tribunal inférieur qui ne peut présenter des garanties objectives d'impartialité en raison du conflit d'intérêts de l'ensemble de ses membres pourrait peut-être être mise en doute. De plus, à la différence de nombreux sujets relevant des tribunaux administratifs, les questions soulevées par le statut juridique des juges des tribunaux hiérarchiquement inférieurs ne présentent pas un degré de spécialisation tel que le tribunal de droit commun ne puisse en traiter.

Une autre modalité de la doctrine de la nécessité concerne le choix du juge chargé de décider du litige. En présence d'un conflit d'intérêts institutionnel, il pourrait parfois être souhaitable qu'un juge extérieur au tribunal soit désigné pour entendre le litige, que ce soit un juge nommé ad hoc ou le membre d'un autre tribunal, en autant que le motif de récusation ne le rende pas luimême inhabile. La désignation de juges suppléants à la Cour fédérale du Canada, dans certains dossiers présentant un conflit d'intérêts institutionnel ${ }^{80}$, allait clairement en ce sens. Bien que ce mécanisme existe aussi dans la législation québécoise, il est limité à la Cour d'appel et ne permet d'y adjoindre que des juges de la Cour supérieure ${ }^{81}$. Une modification législative serait donc nécessaire pour permettre une application plus large de ce mécanisme devant les tribunaux québécois.

80. Addy, supra note 21; Muldoon, supra note 26.

81. L'article $514 \mathrm{du}$ Code de procédure civile permet au juge en chef de la Cour d'appel de demander au juge en chef de la Cour supérieure de lui désigner un ou plusieurs juges de cette cour pour siéger à la Cour d'appel afin d'assurer la bonne expédition des affaires dont elle est saisie. L'application de ce mécanisme a été envisagée dans un cas de conflit d'intérêts : $A$. $c$. B., supra note 6 au para. 32. L'article 12 de la Loi sur les tribunaux judiciaires, L.R.Q. c. T-16, envisage aussi la nomination de juges suppléants à la Cour d'appel, mais seulement en cas de congés, d'absence ou de maladie. 
Un juge d'expérience, rompu par sa carrière à l'exigence d'impartialité, est sans doute le mieux placé pour résister à la pression que produit un conflit d'intérêts. Dans un dossier où l'impartialité des membres de la Cour d'appel fédérale était remise en doute en raison d'un conflit d'intérêts, l'un des juges rappelait ainsi que les trois plus anciens membres du tribunal avaient été désignés pour trancher le litige ${ }^{82}$. Il est cependant difficile d'exercer quelque contrôle extérieur que ce soit à ce sujet. En effet, la prérogative de désigner un juge pour entendre une affaire appartient au juge en chef du tribunal. Les assignations des juges font partie de l'indépendance administrative du tribunal 83 et la jurisprudence a aussi clairement établi qu'on ne peut s'enquérir des raisons qui font en sorte qu'un juge est affecté à un dossier ${ }^{84}$. C'est donc au juge en chef du tribunal qu'incombe la responsabilité de favoriser la plus grande impartialité possible lorsqu'il désigne le juge chargé d'entendre un litige présentant un conflit d'intérêts institutionnel.

Trois aspects de la motivation des jugements rendus dans un contexte de conflit d'intérêts institutionnel méritent plus particulièrement l'attention. Le premier a trait à la divulgation du conflit d'intérêts dans lequel se trouve le juge. Cette pratique, suivie dans certains jugements ${ }^{85}$ et conforme aux règles générales encadrant la récusation ${ }^{86}$, devrait être généralisée par souci de transparence. Qu'on lui demande ou non de se récuser, le juge saisi du litige devrait divulguer la nature et l'étendue de son conflit d'intérêts, particulièrement lorsqu'il est susceptible de tirer un avantage direct du jugement qu'il est appelé à rendre. Certaines circonstances peuvent cependant le dispenser d'une telle divulga-

82. Canada (Minister of Citizenship and Immigration) c. Tobiass, supra note 36 au para. 14.

83. Valente c. La Reine, supra note 57 à la p. 709.

84. MacKeigan c. Hickman, [1989] 2 R.C.S. 796 aux pp. 831-834.

85. À titre d'exemple : Beauregard, supra note 12 aux pp. 549-551.

86. Ainsi, l'article $236 \mathrm{du}$ Code de procédure civile énonce que le juge qui connaît une cause valable de récusation le concernant est tenu, sans attendre qu'elle soit proposée, de la déclarer par un écrit versé au dossier et d'en informer le juge en chef; à ce propos, voir aussi : Conseil canadien de la magistrature, supra note 3 à la p. 46, aux para. E.11 - E.12. 
tion : par exemple, un litige relatif à l'application d'une règle de droit ancienne et bien établie comme l'immunité judiciaire, ou encore un dossier mettant en cause un sujet - comme l'indépendance judiciaire - à propos duquel l'intérêt personnel du juge est évident dans le cadre du litige dont il est saisi.

Le second concerne les raisons qui portent le juge à avoir recours à la doctrine de la nécessité : elles devraient être explicitement énoncées dans le jugement afin, le cas échéant, qu'un tribunal de niveau supérieur puisse apprécier l'opportunité de l'application qui en a été faite. Le troisième aspect se rapporte au mérite du litige : la motivation du jugement devrait être obligatoire. Certaines dispositions du Code de procédure civile imposent aux tribunaux judiciaires de première instance et d'appel l'obligation de motiver leurs jugements ${ }^{87}$. Elles font échec à la règle de common law qui, en principe, n'impose en effet à un tribunal judiciaire aucune obligation de motiver ses décisions ${ }^{88}$. Les motifs de jugement permettent entre autres de vérifier que le tribunal a rendu sa décision suivant la preuve et qu'il a appliqué les règles de droit. Cette fonction de la motivation des jugements est particulièrement importante dans le contexte d'un conflit d'intérêts, puisque tel est précisément aussi l'un des objectifs de l'obligation d'impartialité89. Une même finalité inspire les deux principes, de sorte que l'impossibilité d'assurer pleinement la réalisation de l'un de ces principes - l'exigence d'impartialité - de-

87. Art. 471 et 519 C.p.c.

88. À ce sujet, voir Luc Huppé, Le régime juridique du pouvoir judiciaire, Montréal, Wilson et Lafleur ltée, 2000 aux pp. 132-136. Il existe cependant une obligation de motiver les jugements rendus en matière criminelle : $R$. $c$. Sheppard, [2002] 1 R.C.S. 869.

89. "Je souligne que les principes d'indépendance et d'impartialité visés à l'al. $11 \mathrm{~d})$ ont un double objectif : premièrement, s'assurer que la personne est jugée par un tribunal qui n'est aucunement partial et qui est apte à rendre une décision fondée seulement sur la preuve dont il est saisi, conformément à la loi. Le décideur ne devrait pas être influencé par les parties ni par des forces extérieures, sauf dans la mesure où il est convaincu par les arguments et les plaidoiries portant sur les questions de droit en litige" : $R$. c. Généreux, supra note 59 aux pp. 282-283. 
vrait en toute logique accentuer la portée de l'autre - l'obligation de motivation - afin de favoriser l'atteinte de leur objectif commun.

Dans certains cas, l'appel peut permettre de contrebalancer l'existence d'un conflit d'intérêts au sein du tribunal de première instance, lorsque le conflit d'intérêts n'affecte pas les juges du tribunal d'appel ${ }^{90}$. Le mécanisme d'appel permet alors de corriger tout manquement à l'exigence d'impartialité ayant pu se produire au sein du tribunal subordonné. Les règles déterminant la possibilité de se pourvoir en appel devraient donc recevoir une interprétation souple lorsque le tribunal d'appel ne se trouve pas dans la situation de conflit d'intérêts qui afflige le tribunal de première instance $^{91}$. Il devrait en être de même en ce qui concerne la norme de contrôle utilisée par le tribunal d'appel pour réviser le jugement de première instance rendu par un juge placé dans une situation de conflit d'intérêts institutionnel.

Les institutions judiciaires disposent donc d'une certaine latitude dans l'encadrement des modalités d'application de la doctrine de la nécessité. Outre la détermination des conditions qui permettent d'y avoir recours, plusieurs mesures sont à leur portée pour atténuer les effets, négatifs pour l'apparence d'impartialité du tribunal, qui découlent de l'impossibilité d'empêcher un conflit d'intérêts. Toutefois, d'autres mesures leur échappent et dépendent de la bonne volonté du législateur.

\section{CONCLUSION}

L'existence ponctuelle de situations de conflit d'intérêts institutionnels n'est pas une caractéristique propre aux institutions judiciaires. Les parlementaires qui fixent leur propre rémunération au moyen d'une loi ou qui s'accordent le bénéfice d'une im-

90. Une illustration en est fournie dans les arrêts suivants : The Judges, supra note 10; Canada (Ministre de la Citoyenneté et de l'Immigration) c. Tobiass, supra note 40.

91. Ainsi, lorsque le droit d'appel du jugement est assujetti à une permission préalable du tribunal d'appel, elle devrait en principe être accordée. À titre d'exemple : Gold, supra note 29. 
munité de poursuite dans l'exercice de leurs fonctions, par exemple, se trouvent aussi dans une situation de conflit d'intérêts. Aucune doctrine de la nécessité ne semble nécessaire pour asseoir la légitimité des décisions qu'ils prennent dans de tels contextes.

Le législateur peut certes exercer les pouvoirs qu'il possède, même lorsque les membres des assemblées législatives en sont les bénéficiaires directs. Aucune autre instance ne peut d'ailleurs se substituer à lui pour légiférer à ce propos. En principe, il ne devrait pas en être autrement lorsque les autres institutions de l'État exercent les pouvoirs dont elles sont investies : ces pouvoirs visent l'ensemble des justiciables assujettis à la compétence de l'institution y compris, parfois, ses propres membres.

Si les conflits d'intérêts présentent un aspect particulier au sein de la magistrature, c'est en raison du fait que l'exigence d'impartialité y est plus prononcée que dans les autres institutions, et qu'elle constitue même la qualité première de la fonction judiciaire. C'est pourquoi la doctrine de la nécessité développée par la jurisprudence pour faire face aux situations de conflits d'intérêts doit être appuyée sur des motifs qui prêtent le moins possible à la contestation, et encadrée par des modalités qui en réduisent au maximum les inconvénients. Autant les juges que l'ensemble des justiciables ont intérêt à ce que sa légitimité demeure indiscutable. 
